\title{
Estilo de vida e trabalho de crianças e adolescentes em Programas Saúde da Família
}

\author{
Renata Cristina da Penha Silveira ${ }^{1}$, Maria Lúcia do Carmo Cruz Robazzi ${ }^{2}$
}

\footnotetext{
${ }^{1}$ Enfermeira, Doutora em Enfermagem. Professora Associada da Faculdade de Enfermagem da Universidade Federal de São João Del-Rei. Divinópolis, MG, Brasil. Email: renatasilveira@ufsj.edu.br.

${ }^{2}$ Enfermeira, Doutora em Enfermagem. Professora Titular da Escola de Enfermagem de Ribeirão Preto da Universidade de São Paulo. Ribeirão Preto, SP, Brasil. E-mail: avrmlccr@eerp.usp.br.
}

Recebido: 30/03/2016

Aceito: 09/02/2017.

Publicado: 14/07/2017

Como citar esse artigo:

Silveira RCP, Robazzi MLC. Estilo de vida e trabalho de crianças e adolescentes em Programas Saúde da Família. Rev. Eletr. Enf. [Internet]. 2017 [acesso em: ____;19:a22. Disponível em: http://dx.doi.org/10.5216/ree.v19.40497.

\section{RESUMO}

O objetivo desta investigação foi analisar o estilo de vida e trabalho de crianças e adolescentes cadastrados em Programas de Saúde da Família de cidade do interior paulista. Estudo de abordagem quantitativa, descritiva e transversal, em que crianças e adolescentes foram entrevistados por questionário socioeconômico e laboral e o instrumento Estilo de Vida Fantástico. Os dados foram analisados pelo programa SPSS, versão 19.0. De 168 entrevistados, a maioria era menina (51,8\%), com média de idade de 12,79 anos; parda (50\%); residente em habitações populares $(73,8 \%)$ e sem estudar $(28,5 \%)$; 14\% trabalhavam e 84 famílias recebiam auxílio do Programa Bolsa Família. Quanto ao estilo de vida e trabalho, não houve diferença significativa $(Z=0,87 ; p=0,39)$. O estudo mostrou que mesmo trabalhando, estudando e ajudando os familiares em tarefas domésticas, os entrevistados possuíam tempo para brincar e se divertir e sentir felicidade, indicando que o trabalho não influenciou em seu estilo de vida.

Descritores: Trabalho de Menores; Saúde do Trabalhador; Estilo de Vida; Enfermagem; Estratégia Saúde da Família.

\section{INTRODUÇÃO}

Há 351 milhões de crianças em todo o mundo que trabalham e, ao mesmo tempo, apresentam alterações emocionais, comportamentais e psiquiátricas, que podem ser consideradas um importante problema de saúde pública nos países em desenvolvimento ${ }^{(1)}$. Apesar de existirem estudos relacionados ao trabalho da criança e do adolescente com relativa frequência na literatura científica internacional ${ }^{(2)}$, no Brasil, esse tipo de trabalho representa uma realidade para aproximadamente 3,7 milhões de crianças e adolescentes $^{(3)}$.

Tal trabalho pode favorecer a ocorrência de acidentes e adoecimentos, pois os ambientes laborais são 
construídos para o trabalho de adultos e, muitas vezes, senão na maioria delas, crianças e adolescentes não conseguem se adaptar a estes locais e, tampouco, às suas ferramentas e aos instrumentos característicos do uso por adultos ${ }^{(4)}$.

O fato de máquinas, ferramentas, móveis e equipamentos de proteção individual não terem sido desenhados para uso infanto-juvenil também pode colocar crianças e adolescentes trabalhadores em situação de risco aumentado para desenvolvimento de problemas relacionados ao trabalho ${ }^{(5)}$, que podem alterar o seu estilo de vida e sua saúde.

O estilo de vida pode ser compreendido como uma forma cultural e social de viver e abrange hábitos e condutas capazes de contribuir de forma positiva ou negativa à saúde. Também pode refletir no aumento das taxas de morbidade e mortalidade. O sedentarismo, o consumo de álcool e fumo e a dieta inadequada são exemplos de hábitos que podem aumentar o risco de morte e dos agravos à saúde; por outro lado, uma dieta equilibrada e a atividade física regular são considerados protetores ${ }^{(6)}$.

A existência do trabalho infantil evidencia uma questão grave e séria que acontece no Brasil e no mundo. Por conseguinte, justifica-se este estudo por analisar a relação existente entre o estilo de vida e o trabalho de crianças e suas repercussões nos hábitos infantis, tempo para brincadeiras enquanto crianças.

Diante do exposto até então, o objetivo desta investigação foi analisar o estilo de vida e trabalho de crianças e adolescentes cadastrados em Programas de Saúde da Família.

\section{MÉTODOS}

Estudo de abordagem quantitativa, descritiva e transversal, realizado com crianças e adolescentes pertencentes a famílias cadastradas nos Programas de Saúde da Família (PSF) da Secretaria Municipal de Saúde de um município do interior paulista.

Foi utilizado um questionário com perguntas sobre as características socioeconômicas, tempo para brincadeiras e lazer e caracterização do trabalho e um instrumento do Estilo de Vida Fantástico, validado no Brasil $^{(7)}$.

As 25 questões desse instrumento sobre Estilo de Vida encontram-se dispostas em escala do tipo Likert; 23 possuem cinco alternativas de resposta e duas são dicotômicas. As alternativas estão organizadas em colunas para facilitar sua codificação e a alternativa da esquerda é sempre a de menor relação com o estilo de vida saudável. A codificação das questões é realizada por pontos: zero para a primeira coluna, 1 para a segunda, 2 para a terceira, 3 para a quarta e 4 para a quinta coluna. As questões que só possuem duas alternativas pontuam zero para a primeira coluna e 4 pontos para a última coluna. A soma de todos os pontos permite chegar a um escore total que classifica a criança/adolescente em cinco categorias: "excelente" (85 a 100 pontos) indica que o estilo de vida proporciona ótima influência para a saúde, "muito bom" (70 a 84 pontos) o estilo de vida proporciona adequada influência para a saúde, "bom" (55 a 69 pontos) indica muitos benefícios para a saúde, "regular" (35 a 54 pontos) proporciona algum benefício para a saúde, porém apresenta também riscos e necessita melhorar e de zero a 34 pontos, o estilo de vida apresenta muitos 
fatores de riscos. Quanto menor o escore, maior é a necessidade de mudanças ${ }^{(7)}$.

No presente estudo, a população foi composta por crianças e adolescentes entre 10 e 17 anos dos PSF. Todos os PSF da cidade foram numerados aleatoriamente e listados, e compuseram um banco de dados; a partir daí, foi sorteado um PSF do município por amostragem aleatória simples. Para a coleta de dados, calculou-se amostra de 138 indivíduos com base na incidência de 15\% de crianças e adolescentes que trabalhavam para o segmento de 10 a 17 anos em Ribeirão Preto. Em todas as análises, foi considerado nível de significância de $5 \%(p \leq 0,05)$.

O Instituto Brasileiro de Geografia e Estatística (IBGE) indicou que, em 2010, existiam 2.563 crianças trabalhadoras de 10 a 14 anos; 8.861 crianças de 15 a 17 anos e a população total era de 145.724 ; assim, o percentual de crianças trabalhadoras era de $8 \%{ }^{(8)}$.

A coleta de dados ocorreu de setembro de 2011 a abril de 2013. Primeiramente, realizou-se a revisão manual de todos os prontuários das famílias cadastradas no PSF sorteado, bem como busca por jovens de 10 a 17 anos.

A partir daí, junto dos profissionais do PSF e dos Agentes Comunitários de Saúde (ACS), iniciou-se a coleta de dados com a primeira autora deste estudo, realizando as Visitas Domiciliares (VD) diariamente com o ACS.

A região sorteada para a coleta de dados era vulnerável do ponto de vista de segurança. A presença de traficantes pelas ruas, armas e tráfico de drogas culminou na necessidade do acompanhamento da pesquisadora pelo ACS durante todas as visitas e entrevistas. Estas foram permitidas e realizadas somente no período da manhã e, muitas vezes, houve a necessidade de retornar nos domicílios por não encontrar a criança.

A cada VD e participação dos sujeitos, foi necessária a assinatura do Termo de Consentimento Livre e Esclarecido (TCLE) pelo pai ou a mãe, e pela própria criança e, somente após, ocorreu a aplicação dos questionários.

Após respondidos os questionários, os valores obtidos foram digitados duplamente em banco de dados no programa Excel e, posteriormente, os dados foram analisados pelo Statistical Package for Social Science (SPSS), versão 19.0.

O projeto foi aprovado pelo Comitê de Ética em Pesquisa da Escola de Enfermagem da Universidade de São Paulo, sob protocolo no 1.378/2011 e atendeu as normas brasileiras para pesquisas com seres humanos.

\section{RESULTADOS}

Para se obter uma maior confiabilidade no tamanho da amostra, foram entrevistadas 168 crianças e adolescentes em seus domicílios, sendo adotado o nível de significância $p \leq 0,05$. A Tabela 1 apresenta as características relacionadas ao sexo e à idade dos entrevistados. 
Tabela 1: Análise descritiva das variáveis socioeconômicas e demográficas de crianças e adolescentes entrevistados (n=168). Ribeirão Preto, SP, Brasil, 2013.

\begin{tabular}{|c|c|c|}
\hline Variáveis & $\mathbf{N}$ & $\%$ \\
\hline \multicolumn{3}{|l|}{ Sexo } \\
\hline Feminino & 87 & 51,8 \\
\hline Masculino & 81 & 48,2 \\
\hline \multicolumn{3}{|l|}{ Idade, anos } \\
\hline 10 & 23 & 13,6 \\
\hline 11 & 19 & 11,3 \\
\hline 12 & 34 & 20,2 \\
\hline 13 & 26 & 15,4 \\
\hline 14 & 18 & 10,7 \\
\hline 15 & 18 & 10,7 \\
\hline 16 & 18 & 10,7 \\
\hline 17 & 12 & 7,1 \\
\hline \multicolumn{3}{|l|}{ Cor } \\
\hline Branca & 57 & 33,9 \\
\hline Negra & 22 & 13,1 \\
\hline Parda & 84 & 50,0 \\
\hline Outra & 1 & 0,6 \\
\hline Sem resposta & 4 & 2,4 \\
\hline \multicolumn{3}{|l|}{ Região de residência } \\
\hline Bairro & 44 & 26,2 \\
\hline Habitações populares & 124 & 73,8 \\
\hline \multicolumn{3}{|l|}{ Condição de moradia } \\
\hline Casa própria & 146 & 86,9 \\
\hline Alugada & 21 & 12,5 \\
\hline Sem resposta & 1 & 0,6 \\
\hline \multicolumn{3}{|l|}{ Renda familiar* } \\
\hline Até $\mathrm{R} \$ 1.000,00$ (USD* 497.51) & 48 & 28,5 \\
\hline Acima $\mathrm{R} \$ 1.000,00$ (USD 498.00) & 28 & 16,8 \\
\hline Não responderam & 92 & 54,8 \\
\hline Estuda & 120 & 71,4 \\
\hline Parou de estudar & 48 & 28,5 \\
\hline Total & 168 & 100 \\
\hline
\end{tabular}

* USD: United States Dollar - moeda norte americana, cuja cotação em $1^{\circ}$ de abril de 2013 era de USD 1,00 = R\$ 2,01.

Ao realizar o Teste t para as variáveis "idade" e "sexo" das crianças e adolescentes ( $t=2,31 ; p=0,02)$ houve diferença estatística significativa, mostrando que o sexo feminino possuía escolaridade superior. Para as variáveis "idade" e "escolaridade" utilizou-se o Coeficiente de Correlação de Pearson $(r=0,84 ; p<0,001)$, resultando em coeficiente significativo e positivo, pois quanto maior a idade, maior a escolaridade. Para a análise de "sexo" e "parar de estudar", o Teste Qui Quadrado mostrou que não houve diferença significativa $\left(\chi^{2}=0,95 ; p=0,33\right)$; este mesmo fato (não identificação de diferença estatística significante) foi encontrado em relação a "cor" e "evasão escolar" $\left(\chi^{2}=3,60 ; p=0,17\right)$ e entre as variáveis "crianças e adolescentes trabalhadores" e "receber benefício do governo" $\left(\chi^{2}=1,16 ; p=0,28\right)$.

De 23 crianças e adolescentes trabalhadores, 15 (65,2\%) delas participavam do Programa Bolsa Família (PBF) e destas, 13 trabalhavam e ajudavam no sustento da casa. Das famílias que participavam do PBF, a família com a maior renda possuía somente um filho de 12 anos no sexto ano, já reprovado uma vez por desempenho ruim; a criança ajudava nas tarefas domésticas todos os dias por duas horas e, após, brincava com amigos. 
A família de menor renda residia na região de habitações populares. A criança já fora reprovada duas vezes; tinha dois irmãos (moravam com a avó), ajudava nas tarefas domésticas por uma hora e brincava três dias por semana com os amigos.

A Tabela 2 apresenta dados sobre a evasão escolar.

Tabela 2: Evasão escolar de crianças e adolescentes entrevistados (n=168). Ribeirão Preto, SP, Brasil, 2013.

\begin{tabular}{ccc}
\hline Variáveis & $\mathbf{n}$ & $\mathbf{\%}$ \\
\hline Meninos estudantes & 55 & 67,9 \\
Meninas estudantes & 65 & 74,7 \\
Meninos que pararam de estudar & 26 & 32,1 \\
Meninas que pararam de estudar & 22 & 25,3 \\
\hline Total & $\mathbf{1 6 8}$ & $\mathbf{1 0 0}$ \\
\hline
\end{tabular}

Ao realizar o Teste Qui Quadrado para "sexo" e a variável "pararam de estudar", não houve diferença estatística significativa $\left(\chi^{2}=0,95 ; p=0,33\right)$. Dos 168 entrevistados, $48(28,6 \%)$ pararam de estudar. Para analisar a relação entre as variáveis "pararam de estudar" e "idade", realizou-se o Teste não paramétrico de Mann-Whitney $(Z=4,47 ; p<0,001)$, obtendo-se diferença significativa, pois os que pararam de estudar possuíam idade significativamente superior.

A Tabela 3 apresenta, a seguir, a análise descritiva das crianças e adolescentes trabalhadoras.

Tabela 3: Análise descritiva de crianças e adolescentes trabalhadoras entrevistados ( $n=23$ ). Ribeirão Preto, SP, Brasil, 2013.

\begin{tabular}{|c|c|c|}
\hline Variáveis & $\mathbf{n}$ & $\%$ \\
\hline \multicolumn{3}{|l|}{ Trabalho } \\
\hline Sim & 23 & 13,6 \\
\hline \multicolumn{3}{|l|}{ Idade início no trabalho, anos } \\
\hline 8 & 1 & 0,6 \\
\hline 9 & 1 & 0,6 \\
\hline 10 & 1 & 0,6 \\
\hline 11 & 1 & 0,6 \\
\hline 13 & 2 & 1,2 \\
\hline 14 & 5 & 3,0 \\
\hline 15 & 3 & 1,8 \\
\hline 16 & 7 & 4,2 \\
\hline 17 & 2 & 1,2 \\
\hline \multicolumn{3}{|l|}{ Pagamento } \\
\hline Recebia pelo trabalho & 21 & 12,5 \\
\hline Não recebia pelo trabalho & 2 & 1,2 \\
\hline Sem resposta & 145 & 86,3 \\
\hline \multicolumn{3}{|l|}{ Quanto recebia* } \\
\hline De USD 2,48-50 & 3 & 13,0 \\
\hline De USD 51-250 & 13 & 56,5 \\
\hline De USD 251-500 & 5 & 21,8 \\
\hline Não informou valores & 2 & 8,7 \\
\hline \multicolumn{3}{|l|}{ Auxílio na despesa familiar } \\
\hline Não auxiliava seus pais & 4 & 17,3 \\
\hline Auxiliava seus pais & 19 & 82,3 \\
\hline Total & 23 & 100 \\
\hline
\end{tabular}

* USD: United States Dollar - moeda norte americana, cuja cotação em $1^{\circ}$ de abril de 2013 era de USD $1,00=\mathrm{R} \$ 2,01$.

Os jovens entrevistados realizavam trabalhos como ajudante de pedreiro, vendedor de balas e doces 
na rua, carregador de mudanças, pintor, vendedor de sorvetes, dentre outros. Dos 23 trabalhadores, 15 $(65,2 \%)$ após o trabalho ainda ajudavam os pais nas tarefas da casa por mais uma a três horas, lavando a louça, o banheiro, arrumando a casa, cuidando do irmão mais novo, fazendo comida, dentre outras tarefas.

Para as variáveis relacionadas a "idade de início no trabalho" e "horas trabalhadas por dia", foi utilizado o Coeficiente de Correlação de Spearman ( $r=0,55 ; p=0,01)$, coeficiente significativo e positivo, ou seja, quanto maior a idade que começou a trabalhar, mais horas trabalhava diariamente; a mesma situação aconteceu em relação ao "trabalho" da criança e o "pagamento pelo trabalho" em que esse Coeficiente mostrou-se significativo e positivo, pois quanto mais a criança recebia, mais ela ajudava a família financeiramente $(r=0,50 ; p=0,04)$.

A Tabela 4 apresenta as atividades de lazer e brincadeiras dos entrevistados.

Tabela 4: Descrição das atividades de lazer e brincadeiras de crianças e adolescentes entrevistados ( $n=168)$. Ribeirão Preto, SP, Brasil, 2013.

\begin{tabular}{lcc}
\hline & Variáveis & $\mathbf{n}$ \\
\hline Lazer & 158 & 94,0 \\
Sim & 9,4 \\
Não & 1 & 0,6 \\
Sem resposta & \\
Tempo para brincadeiras & 105 \\
Todos os dias & 19 \\
Duas vezes na semana & 62,5 \\
Três vezes na semana & 11,3 \\
Algumas horas e, após, ajudava os pais nas tarefas de casa & 3,5 \\
Não brincava & 29 \\
Sentimento de felicidade & 9 & 17,3 \\
Considerava-se muito feliz & 5,4 \\
Considerava-se infeliz & 162 \\
\hline
\end{tabular}

Em relação às atividades de lazer e brincadeiras, a maioria dos entrevistados informou que as realizava diariamente, mas também, ajudava os pais em tarefas domésticas.

Em relação à "felicidade" e à "condição de vida" $(p=0,62)$, não houve diferença significativa no Teste Qui Quadrado. Por meio do Teste não paramétrico de Mann-Whitney para comparar "estilo de vida" e "estudar" $(Z=3,12 ; p=0,002)$ houve diferença estatística significativa, ou seja, quem estudava possuía estilo de vida significativamente diferenciado daqueles que pararam de estudar. Por meio do Teste t para amostras independentes do "estilo de vida" e "sexo" ( $t=0,85 ; p=0,40)$, constatou-se que não houve diferença significativa.

Ao realizar a análise do "estilo de vida" das crianças e o "local de moradia", utilizou-se o Teste não paramétrico de Mann-Whitney para a comparação encontrando-se diferença significativa $(Z=3,02 ; p=0,003)$, ou seja, as crianças que moravam em bairros possuíam estilo de vida significativamente superior às que residiam em habitações populares; tinham maior acesso às atividades de recreação, alimentação, cultura, lazer e atividades físicas.

Em relação ao "estilo de vida" e "trabalho" dos entrevistados utilizou-se o Teste não paramétrico de 
Mann-Whitney para a comparação, constatando-se que não houve diferença significativa $(Z=0,87 ; p=0,39)$, pois o trabalho não influenciou no estilo de vida das crianças e adolescentes.

\section{DISCUSSÃO}

Segundo a Organização das Nações Unidas (ONU) e o Fundo das Nações Unidas para a Infância (UNICEF), ocorreu um decréscimo no número de crianças no trabalho a partir de 1995. Em 2006, 5,3 milhões; em 2007, 4,8 milhões; em 2008, 4,5 milhões e em 2009, 4,2 milhões $^{(9)}$. Esses dados coincidem com os da presente pesquisa e com a literatura ${ }^{(10-12)}$. Apesar de haver a diminuição do trabalho infanto-juvenil, ainda existem crianças e adolescentes trabalhando e suprimindo-se de determinados privilégios existentes somente na infância e adolescência, como brincadeiras e estudo.

Em relação ao sexo e idade, investigação realizada em Minas Gerais objetivou caracterizar o trabalho realizado por crianças e adolescentes de uma escola estadual e a possível evasão escolar dos mesmos; identificou que de 56 alunos matriculados no Ensino Fundamental e Médio dessas escolas, 53 trabalhavam e 62,2\% eram meninas. Em relação à idade dos entrevistados, variou de 10 a 17 anos; $46 \%$ se autodeclararam pardos, $37,7 \%$ brancos e $17 \%$ negros $^{(12)}$. Estudo que objetivou investigar as características educacionais e sociodemográficas de jovens brasileiros trabalhadores e não trabalhadores de sete capitais brasileiras, identificou $54,2 \%$ dos participantes como do sexo feminino e a idade média, entre jovens trabalhadores e não trabalhadores, foi de $16,1^{(13)}$.

Corroborando os resultados desta investigação, pesquisa realizada em João Pessoa (PB) com 398 crianças e adolescentes estudantes de escolas públicas municipais e estaduais, de sete e 18 anos de idade, mostrou que 67\% eram afrodescendentes (negros, mulatos, pardos); 30\% eram brancos e 3\% amarelos; 54,2\% trabalhavam. Destes, $29,15 \%$ trabalhavam fora e $25,13 \%$ realizavam trabalho doméstico. Os trabalhadores domésticos tinham entre 10 a 18 anos, 34\% entre 10 a 14 anos e $25 \%$ entre 15 e 16 anos; $73 \%$ da amostra era do sexo feminino ${ }^{(14)}$.

A maioria de crianças e adolescentes trabalha informalmente e inicia suas atividades laborais precocemente $^{(10)}$.

Importante destacar que os que estudavam iniciam-se precocemente no mercado de trabalho para ajudar na renda familiar e no sustento da família, fato também encontrado na literatura. Ao chegar em casa, ao invés de descansar, estudar ou brincar, essas crianças e adolescentes precisam ajudar os pais nas tarefas domésticas, que os deixam cansados e fazem com que abandonem os estudos ${ }^{(10)}$. Nesta investigação, a maioria das crianças que interrompeu os estudos para trabalhar fora de casa e ajudar os pais nas despesas da casa era constituída por meninos.

Dentre crianças e adolescentes trabalhadores em ruas, 75\% eram meninas, a maioria entre nove a 17 anos, com jornada de trabalho de duas a 12 horas diárias, evasão escolar em 5\%. Houve significância estatística entre a variável "idade" e "escolaridade" e o "atraso escolar" ( $p=0,001)$ em mais de 70\% dos casos. Em $54 \%$ das crianças, as principais razões para iniciarem no trabalho foram devido à complementação da 
renda familiar e $70 \%$ recebiam auxílio de programas governamentais ${ }^{(15)}$.

Sobre o início precoce no mercado de trabalho, estudo com o objetivo de descrever a prevalência de trabalho em estudantes da nona série do Ensino Fundamental de escolas públicas e privadas brasileiras teve como resultados que 52,2\% eram do sexo feminino, 63,4\% tinham entre 14 e 15 anos de idade, $82,8 \%$ estudavam em escolas públicas, $13,1 \%$ relataram ter algum trabalho e $8,9 \%$ trabalhavam recebendo remuneração ${ }^{(4)}$.

Várias razões podem levar à inserção precoce de crianças e adolescentes no mercado de trabalho, mas o principal motivo apontado pela maioria dos estudos econômicos refere-se à baixa renda familiar. Assim, a oferta da mão de obra das crianças é explicada como consequência da queda do consumo abaixo de um nível mínimo desejado pela família, que pode ocorrer em maior ou menor grau, dependendo do período do ciclo familiar ${ }^{(16)}$.

No presente estudo, de 23 crianças trabalhadoras, 65,2\% participavam do Programa Bolsa Família (PBF); destas, 13 trabalhavam e ajudavam no sustento da casa.

Tais achados constatam importâncias contrárias àquelas do PBF; esse benefício é composto por duas linhas de pobreza: renda familiar per capita, soma de todas as rendas de todos os membros da família, dividida pelo número de membros; famílias cuja renda per capita for inferior à linha de pobreza extrema têm direito a um benefício fixo, independente do número de pessoas na família. Todas as crianças e adolescentes entre seis e 15 anos devem estar devidamente matriculadas e com frequência escolar mensal mínima de $85 \%$, os estudantes entre 16 e 17 anos devem ter frequência de, no mínimo, $75 \%$ e não trabalhar ${ }^{(17)}$.

É importante enfatizar que o trabalho expõe crianças e adolescentes aos riscos físicos (ruído e temperatura), biológicos (bactérias, vírus, fungos), químicos (agrotóxicos, poeiras, solventes e tintas) ${ }^{(5)}$, ergonômicos e psicossociais ${ }^{(18)}$, aliados à jornada e ritmos de trabalho, relações de poder ${ }^{(5)}$, além de alterações na saúde, exposição aos acidentes de trabalho e doenças ${ }^{(10)}$.

Uma investigação com o objetivo de descrever as características de acidentes de trabalho em jovens canadenses constatou que o sexo masculino foi o mais acometido $(63,9 \%)$ por queimaduras, esmagamento, amputações, ferimentos elétricos, feridas abertas e lesões oculares ${ }^{(19)}$.

Em estudo realizado no interior de São Paulo com o objetivo de identificar as repercussões à saúde em decorrência do trabalho com 117 adolescentes, 44 relataram possuir alterações na saúde. Destes, 76,2\% relataram dor em diversos segmentos do corpo (dor em membros por $20,7 \%$, cefaleia por $27,2 \%$, dorsalgia por $18,5 \%$, mialgia por $2,2 \%$, dentre outros) $)^{(20)}$.

Sobre os riscos psicossociais aos quais essas crianças e adolescentes trabalhadores ficam expostos, investigação ${ }^{(4)}$ constatou que as proporções de estudantes que relataram ter tido dificuldade para dormir nos últimos 12 meses $(16,1 \%)$ foram mais elevadas entre os que trabalhavam.

Dessa forma, ao iniciar-se no trabalho precocemente, crianças e adolescentes deixam de estudar para trabalhar e ajudar nas despesas da família, o que reduz a sua escolarização, aumenta sua evasão escolar e torna-os mão de obra barata e desqualificada no futuro ${ }^{(10)}$. 
Neste estudo foi possível detectar que muitas crianças trabalhavam, estudavam e ajudavam os pais em tarefas domésticas e que a maioria informou que possuía tempo para brincar e se divertir (94\%) e sentiase feliz $(97,6 \%)$, indicando que o trabalho não influenciou em seu estilo de vida.

Brincar é uma necessidade da criança; é o meio pelo qual ela se desenvolve nos aspectos físicos, emocionais, cognitivos e sociais. Os brinquedos estimulam o desenvolvimento e a aprendizagem da criança. Cabe ao adulto propiciar condições para que as crianças brinquem ${ }^{(21)}$.

Outro fator relevante relacionado aos entrevistados referiu-se aos $97,6 \%$ deles que, apesar de residirem em habitações populares, enfrentando condições de pobreza ou extrema pobreza, alguns, incentivados a trabalhar para ajudar no sustento da família, informaram estar felizes com o seu estilo de vida e não gostariam de mudar.

Em contraposição, crianças e adolescentes que não trabalhavam, ao serem questionados sobre o que gostariam que melhorasse em suas vidas, disseram que queriam estudar mais para progredir na vida; ter sua própria casa; comprar comida e até mesmo conseguir trabalho para ajudar a família.

Sabe-se que a inserção no mercado de trabalho precocemente proporciona uma falsa realidade de que o ganho da criança ou adolescente irá ajudar nas despesas da família e, consequentemente, haverá melhora econômica. No entanto, é sabido que iniciar esse trabalho deixa a criança cansada, desestimulada com os estudos e a infância ${ }^{(4-5,10-12,20,22)}$. Em consequência, pode abandonar os estudos e perpetuar o círculo de pobreza da família tornando-se mão de obra desqualificada e barata com estilo de vida ruim.

Nesse sentido, frequentar a escola, assistir às aulas e realizar as tarefas escolares foram apontados como fatores decisivos para conseguir um trabalho melhor no futuro e alcançar condições de vida mais dignas $^{(13)}$, além de estilo de vida saudável.

\section{CONCLUSÃO}

O objetivo do estudo foi analisar o estilo de vida e trabalho de crianças e adolescentes cadastrados em Programas de Saúde da Família. Na comparação entre o estilo de vida e o trabalho desses jovens não houve diferença estatística significativa. A comparação entre o estilo de vida e o estudo teve diferença estatística significativa. Crianças e adolescentes que estudavam possuíam estilo de vida significativamente diferenciados em relação aos que pararam de estudar, em relação a moradia, alimentação, atividades de lazer e cultura junto aos seus familiares. 0 mesmo ocorreu com a análise entre o estilo de vida e local de moradia, constatando que houve diferença estatística significativa, ou seja, as crianças e adolescentes que moravam no bairro possuíam estilo de vida significativamente superior às que residiam em habitações populares.

Em relação as limitações do estudo destaca-se a região sorteada para a coleta de dados, ou seja, uma região extremamente vulnerável do ponto de vista de segurança. Houve necessidade do acompanhamento por uma ACS durante todas as visitas e entrevistas que ocorreram no período da manhã e, muitas vezes, houve a necessidade de retornar nos domicílios por não encontrar a criança, o que pode ser considerado um 
fator limitante para se obter uma amostra mais significativa.

O estudo avança no conhecimento na área da saúde pois mostrou que mesmo trabalhando, estudando e ajudando os familiares em tarefas domésticas, os jovens entrevistados possuíam tempo para brincar, se divertir e estarem felizes como crianças, ou seja, o trabalho não influenciou em seu estilo de vida.

É necessário ampliar esse tema de estudo junto aos profissionais de saúde e educadores, para propor orientações que impeçam o trabalho infantil e mantenham as crianças e adolescentes somente na escola. Enfermeiros precisam orientar sua equipe a observar e avaliar integralmente todas as famílias visitadas em relação a presença do trabalho infantil, não atendo-se, essencialmente, às ações curativas, reposição de medicações e agendamentos. Orientações periódicas do enfermeiro nas escolas podem ser de grande valia, já que o professor bem orientado e informado sobre esse tipo de trabalho pode observar diariamente o comportamento da criança em relação a cansaço, sono, tarefas escolares realizadas, faltas e questioná-la e a família sobre tais comportamentos, visando buscar soluções para impedir a evasão escolar e o trabalho infanto-juvenil.

Por outro lado, tem-se, além disso, a importância da escola na vida da criança e do adolescente em introduzir a cultura e a cidadania, além de auxiliá-los na construção da autonomia ao prepará-los, através da educação, para exigências do mercado de trabalho, que exige mão de obra qualificada. É evidente a necessidade de equipe multidisciplinar para identificar e promover ações de prevenção ao trabalho infantil.

\section{Agradecimento e financiamento}

Ao Conselho Nacional de Desenvolvimento Científico e Tecnológico (CNPQ) pelo financiamento dessa pesquisa (Programa Nacional de Pós-Doutorado, PNPD, Processo n: 561018/2010-3).

\section{REFERÊNCIAS}

1. Al-Gamal E, Hamdan-Mansour AM, Matrouk R, Nawaiseh MA. The psychosocial impact of child labour in Jordan: a national study. Int J Psychol [Internet]. 2013 [cited 2016 out 7];48:1156-64. Available from:

http://dx.doi.org/10.1080/00207594.2013.780657.

2. Cabral ES, Sena RR. Desgastes na saúde de jovens que trabalham e estudam: uma revisão integrativa. Nursing. 2016;17(222):1262-5.

3. Dias JC, Araújo GS. O trabalho infantil doméstico no Brasil: avaliação a partir dos microdados da Pnad/IBGE (20082011). Brasília DF: Fórum Nacional de Prevenção e Erradicação do Trabalho Infantil; 2013.

4. Giatti L, Campos MO, Crespo CD, Andrade SSCA, Barreto SM. Labor in early life, vulnerability for health in Brazilian schoolchildren: National Adolescent School-based Health Survey (PeNSE 2012). Rev. bras. epidemiol. [Internet]. 2014 [citado 2016 Dez 12];17 supl 1:17-30. Available from: http://dx.doi.org/10.1590/1809-4503201400050003. 5. Rozenberg R, Tendrih L. Adolescentes no Rio de Janeiro: educação, trabalho e riscos à saúde. Adolesc Saude [Internet]. 2007 [cited 2016 out 7];4(3):33-6. Available from:

http://adolescenciaesaude.com/detalhe_artigo.asp?id=97

6. Ferreira DK, Bonfim C, Augusto LG. [Factors associated with the lifestyle of military police officers]. Ciência da Saúde Coletiva [Internet]. 2011 [cited 2016 out 7];16(8):3403-12. Available from:

https://www.researchgate.net/profile/Lia_G_da_S/publication/51589643 Factors associated_with_the lifestyle_of military_police_officers/links/54cfa7bc0cf24601c0951ebb.pdf 
7. Añez CR, Reis RS, Petroski EL. Versão brasileira do questionário "estilo de vida fantástico": tradução e validação para adultos jovens. Arq Bras Cardiol [Internet]. 2008 [cited 2016 out 7];91(2):102-9. Available from:

http://www.scielo.br/scielo.php?script=sci_arttext\&pid=S0066-782X2008001400006\&lng=en

8. Instituto Brasileiro de Geografia e Estatística (IBGE). Censo Demográfico 2010 [Internet]. Brasília, DF: Ministério das Cidades: 2016 [cited 2016 out 7]. Available from:

http://www.cidades.ibge.gov.br/xtras/temas.php?lang=\&codmun=354340\&idtema=117\&search=sao-paulo| ribeiraopreto|ensino-matriculas-docentes-e-rede-escolar-2012

9. Organização das Nações Unidas (ONU); Fundo das Nações Unidas Para a Infância (UNICEF). Raio X sobre a situação dos direitos humanos no Brasil. Brasília, DF: ONU/UNICEF; 2008.

10. Silveira RC, Ribeiro LC, Secco IA, Robazzi ML. Caracterización socioeconómica y demográfica de niños y adolescentes que estudian y trabajan simultáneamente fuera de casa. Rev Esc Enferm USP [Internet]. 2012 [cited 2016 out 7];46(2):280-6. Available from: http://www.revistas.usp.br/reeusp/article/view/40946/44459

11. Cecilio SG, Salles JB, Robazzi ML, Silveira RC. Trabalho de crianças e adolescentes e suas consequências: uma revisão integrativa da literatura. Nursing (São Paulo). 2012;14(168):250-5.

12. Cecilio SG, Silveira RC. Caracterização do trabalho de menores de uma escola estadual de Divinópolis-MG. Ciencia y Enfermeria [Internet]. 2014 [cited 2016 out 7];20(1):47-60. Available from:

http://www.scielo.cl/scielo.php?script=sci_arttext\&pid=S0717-95532014000100005\&lng=es.

13. Thomé LD, Pereira AS, Koller SH. O Desafio de Conciliar Trabalho e Escola: Características Sociodemográficas de Jovens Trabalhadores e Não-trabalhadores. Psicologia: Teoria e Pesquisa [Internet]. 2016 [cited 2016 out 7];32(1):1019. Available from: https://dx.doi.org/10.1590/0102-37722016011944101109

14. Lima MB, Ribeiro DF, Andrade AS. Percepções de crianças e adolescentes sobre seu trabalho informal. Psicologia em Revista [Internet]. 2011 [cited 2016 out 7];17(1);48-66. Available from:

http://pepsic.bvsalud.org/scielo.php?script=sci_arttext\&pid=S1677-11682011000100003\&lng=pt\&tlng=pt

15. Meneses RB, Cardoso RC, Guimaraes AG, Goes JA, Silva AS, Argolo SV. O comércio de queijo de coalho na orla de Salvador, Bahia: trabalho infantil e segurança de alimentos. Rev Nutr [Internet]. 2012 [cited 2016 out 7]; 25(3):381-92. Available from: https://repositorio.ufba.br/ri/bitstream/ri/15584/1/Roberta\%20Barbosa\%20de\%20Meneses.pdf 16. Ferreira-Batista N, Cacciamali MC. Migração familiar, trabalho infantil e ciclo intergeracional da pobreza no estado de São Paulo. Nova Econ [Internet]. 2012 [cited 2016 out 7];22(3):515-54. Available from:

http://www.scielo.br/scielo.php?pid=S0103-63512012000300004\&script=sci_arttext\&tlng=es

17. Soares S, Sátyro N. O programa bolsa família: desenho institucional, impactos e possibilidades futuras. Texto para discussão n 1424. IPEA. Brasilia, 2009. acessos em 12 dez. 2016. Disponível em

http://www.en.ipea.gov.br/agencia/images/stories/PDFs/TDs/td_1424.pdf

18. Caran VC, Freitas FC, Alves LM, Pedrão LJ, Robazzi ML. Riscos ocupacionais, psicossociais e sua repercussão na saúde de docentes universitários. Rev Enferm UERJ. [Internet]. 2011 [cited 2016 out 7];19(2):255-61. Available from: http://www.facenf.uerj.br/v19n2/v19n2a14.pdf

19. Pratt B, Cheesman J, Breslin C, Do MT. Occupational injuries in Canadian youth: an analysis of 22 years of surveillance data collected from the Canadian Hospitals Injury Reporting and Prevention Program. Health Promot Chronic Dis Prev Can. 2016;36(5):89-98.

20. Pimenta AA et al. Repercussões do trabalho na saúde dos adolescentes trabalhadores. Acta paul. enferm. 2011; 24(5): 701-716. Disponível em http://dx.doi.org/10.1590/S0103-21002011000500017.

21. Silva LF, Cabral IE. O resgate do prazer de brincar da criança com câncer no espaço hospitalar. Rev. Bras. Enferm 2015; 68(3):391-397. Disponível em: http://dx.doi.org/10.1590/0034-7167.2015680303i.

22. Tempestti C, Alfaro S, Cappelen L. Estilos de vida y hábitos alimentarios en adolescentes escolarizados de poblaciones urbanas y rurales, 2010, Salta. Actual Nutr [Internet]. 2013 [cited 2016 out 3];14(2):133-40. Available from: http://www.revistasan.org.ar/pdf_files/trabajos/vol_14/num_2/RSAN_14_2_133.pdf 